\title{
Fractional Euler-Bernoulli Beam Theory Based on the Fractional Strain-Displacement Relation and its Application in Free Vibration, Bending and Buckling Analyses of Micro/Nanobeams
}

\author{
Zaher RAhimi $^{a, *}$, SAmrand Rash Ahmadi ${ }^{a}$ And W. Sumelka ${ }^{b}$ \\ ${ }^{a}$ Mechanical Engineering Department, Urmia University, Urmia, Iran \\ ${ }^{b}$ Poznan University of Technology, Institute of Structural Engineering, Piotrowo 5, 60-965 Poznan, Poland
}

(Received May 12, 2018; in final form July 4, 2018)

Applications of fractional calculus in the constitutive relation lead to the fractional derivatives models. They are stately generalization of the integer derivatives models - this general form makes fractional derivatives models more flexible and suitable to describe properties and behavior of different materials/structures. In the present work, the general strain deformation gradient has been presented by using the modified conformable fractional derivatives definition. Within this approach the fractional Euler-Bernoulli beam theory has been formulated and applied to the analysis of free vibration, bending and buckling of micro/nanobeams which exhibit strong scale effect.

DOI: 10.12693/APhysPolA.134.574

PACS/topics: fractional derivatives model, fractional Euler-Bernoulli theory, free vibration, bending, buckling, nanobeam

\section{Introduction}

Fractional calculus has become an exciting new mathematical method of solution of diverse problems in mathematics, science, and engineering [1-3]. Recent advances of fractional calculus are dominated by modern examples of applications in differential and integral equations, physics, signal processing, fluid mechanics, viscoelasticity, mathematical biology and electrochemistry [4].

Applications of fractional calculus have been brought to applied mechanics' problems and leads to fractional derivatives models (FDMs). Many authors pointed out that derivatives and integrals of non-integer order are very suitable for the description of properties of various real materials [5]. These models are the generalized form of classical models, therefore, they are able to describe the behavior of materials better than integer derivatives models (IDMs). For instance, Challamel et al. [6] by using fractional derivatives (FDs) generalized the Eringen nonlocal theory and showed that the optimized fractional derivative model has a perfect matching with the dispersive wave properties of the Born-Kármán model of lattice dynamics and is better than the classical Eringen theory. Also Demir et al. [7] studied vibration of viscoelastic beam that obeys a fractional differentiation constitutive law and stated that FDs are useful for describing the occurrence of vibrations in engineering practice. FDMs have been introduced in different problems, Sumelka [8] applied fractional calculus to a classical problem of the

*corresponding author; e-mail: st_z.rahimi@urmia.ac.ir structural mechanics and introduced nonlocal Kirchhofflove plate theory. Atanackovic and Stankovic [9] modified the kinematics strain-displacement relationship with an alternative nonlocal formulation and by using the Caputo fractional derivatives generalized wave equation in nonlocal elasticity. Lazopoulos [10] assumed that strain energy density depends not only on the local strain but also on a fractional derivative of the strain. Carpinteri et al. [11] by means of attenuation function of strain and the Caputo fractional derivatives introduced a fractional calculus approach to the nonlocal elasticity.

In the present work, the general strain deformation gradient has been presented by using modified conformable fractional derivatives definition (CFDD). The fractional Euler-Bernoulli beam theory (FEBBT) has been presented based on this general form and has two free parameters: fractional parameter (which control the displacement's derivative in strain-displacement relation) and length scale parameter to consider size effects in the micron and the sub-micron scales. Finally, vibration of clamped-clamped (C-C) microbeams under axial force and free vibration, bending and buckling of nanobeam have been studied by the theory. It should be emphasized that compared to the previous papers [12-14] the novelty lays in the definition of the constitutive relation which is based on the fractional strain, as mentioned, whereas in $[12,13]$ the constitutive relation is based on the general form of the Eringen nonlocal elasticity theory, and in [14] the formulation is based on the fractional strain energy.

This paper has been divided into four sections: 1 . Using fractional Taylor series expansion and the CFDD, the general form of the deformation gradient has been introduced. 2. The FEBBT based on the general form of 
the strain has been presented which has two free parameters: fractional parameter to control the displacement derivative in the constitutive relation and the length scale parameter to consider the size effects in micron and sub-micron scales. 3. To demonstrate the functionality of FEBBT the non-dimensional frequency of the microbeams under axial force has been compared with the experimental data and EBT. 4. Bending, buckling and vibration of nanobeams has been studied based on the FEBBT.

\section{Basic definitions and tools}

In this section, the basic definitions that are necessary for the theory are introduced namely the fractional derivatives definition and the fractional Taylor series expansion.

\subsection{Modified conformable fractional derivatives definition}

Modified CFDD is a definition, which has been presented by Khalil et al. [15] and Tallafha et al. [16] is:

Let $f, g: R \rightarrow R$. Then modified CFDD is

$$
\mathrm{D}^{\alpha}(f)(x)=\frac{\partial^{\alpha} f}{\partial x^{\alpha}}=|x|^{(\lceil\alpha\rceil-\alpha)} \frac{\mathrm{d}^{\lceil\alpha\rceil} f(x)}{|\mathrm{d} x|^{\lceil\alpha\rceil}} .
$$

According to the modified CFDD, its application to the multi-variable functions (Appendix A) reads:

$$
\begin{aligned}
& \mathrm{D}_{x}^{\alpha}(g)(x, y)=\frac{\partial^{\alpha} g}{\partial x^{\alpha}}=|x|^{(\lceil\alpha\rceil-\alpha)} \frac{\partial^{\lceil\alpha\rceil} g(x, y)}{|\partial x|^{\lceil\alpha\rceil}}, \\
& \mathrm{D}_{y}^{\alpha}(g)(x, y)=\frac{\partial^{\alpha} g}{\partial y^{\alpha}}=|y|^{(\lceil\alpha\rceil-\alpha)} \frac{\partial^{\lceil\alpha\rceil} g(x, y)}{|\partial y|^{\lceil\alpha\rceil}},
\end{aligned}
$$

where $\alpha \in(0, \infty), f$ is $(n+1)$-differentiable at $x>0$ and $\lceil\alpha\rceil$ is the smallest integer greater than or equal to $\alpha$.

\subsection{Fractional Taylor series expansion}

Fractional Taylor series expansion (FTSE) is a generalization of the one presented in [17].

Assume that $f$ is an infinitely $\alpha$-differentiable function, for some $0<\alpha \leq 1$ at a neighborhood of a point $x_{0}$. Then $f$ has the fractional power series expansion in the form

$$
\begin{aligned}
& f(x)=\sum_{0}^{\infty} \frac{\left(D_{x_{0}}^{\alpha} f\right)^{(k)}\left|\left(x-x_{0}\right)\right|^{k \alpha}}{\alpha^{k} k !} \\
& x_{0}-R^{1 / \alpha}<x<x_{0}+\mathrm{R}^{1 / \alpha}, \quad R>0,(3)
\end{aligned}
$$

where $\left(\mathrm{D}_{x_{0}}^{\alpha} f\right)^{(k)}$ means the application of the fractional $k$ times derivative.

\section{General form of strain in terms of fractional calculus}

The general form of strains is obtained by generalization of the displacement derivatives in the straindisplacement relation, namely the first derivatives are substituted with derivatives of order $\alpha$. Parameter $\alpha$ can be integer and non-integer number between 0 and $1(0<\alpha \leq 1)[9]$ and leads to the appearance of the fractional calculus in the equation of the motion.

The general form of strain presented already by Sumelka [8, 18] and Atanackovic and Stankovic [9] where based on the Riesz-Caputo definition in which the integral form makes the numerical solution of the governing equation difficult. In the present work, the general form has been presented based on modified CFDD and unlike previous works, its usability has been studied from two part of view: 1. geometrical view, by using fractional Taylor series expansion method, 2. continuum mechanics view. In addition, the use of the modified CFDD makes the numerical solution of the governing equation simpler.

\subsection{Geometrical derivation of the general strains}

FDs allow us to make a generalization of integer derivatives, and therefore in this part by using FTSE we present a general form of deformation gradient and improve the strain tensor by assuming a general form as below.

Consider a two-dimensional deformation of an infinitesimal rectangular material element with dimensions $\mathrm{d} x^{\alpha}$ by $\mathrm{d} y^{\alpha}$ where $0<\alpha \leq 1$ but with this proviso that value of alpha satisfies the small deformation theory. It means that the value of $\alpha$ must not be too small - after the deformation rectangular material element should take the form of a rhombus. Based on small deformation theory and fractional Taylor series expansion we have: $u(x+\mathrm{d} x, y) \approx u(x, y)+\frac{1}{\alpha} \frac{\partial^{\alpha} u}{\partial x^{\alpha}}|\mathrm{d} x|^{\alpha}$ with similar expansions for all other terms - for $\alpha=1$ the classical form is obtained of course.

Next, based on Eq. (1):

$$
u(x+\mathrm{d} x, y) \approx u(x, y)+\frac{1}{\alpha}\left(|x|^{1-\alpha} \frac{\partial u}{\partial x}|\mathrm{~d} x|^{\alpha}\right),
$$

so from the geometry of the deformed element $\left(\frac{1}{\alpha} \frac{\partial^{\alpha} v}{\partial x^{\alpha}}|\mathrm{d} x|^{\alpha} \ll 1\right)$ we have

$$
\begin{gathered}
A^{\prime} B^{\prime}=\sqrt{\left(|\mathrm{d} x|^{\alpha}+\frac{1}{\alpha} \frac{\partial^{\alpha} u}{\partial x^{\alpha}}|\mathrm{d} x|^{\alpha}\right)^{2}+\left(\frac{1}{\alpha} \frac{\partial^{\alpha} v}{\partial x^{\alpha}}|\mathrm{d} x|^{\alpha}\right)^{2}} \approx \\
\left(1+\frac{1}{\alpha} \frac{\partial^{\alpha} u}{\partial x^{\alpha}}\right)|\mathrm{d} x|^{\alpha} .
\end{gathered}
$$

Note that when $\alpha \rightarrow 0$ then $\frac{1}{\alpha} \frac{\partial^{\alpha} v}{\partial x^{\alpha}}|\mathrm{d} x|^{\alpha}$ or $\frac{1}{\alpha} \frac{\partial^{\alpha} x}{\partial x^{\alpha}}|\mathrm{d} x|^{\alpha} \rightarrow$ $\infty\left(\frac{1}{\alpha} \frac{\partial^{\alpha} v}{\partial x^{\alpha}}|\mathrm{d} x|^{\alpha}<<1\right)$ since $0<\alpha \leq 1$.. The normal strain in the $x$-direction of the rectangular element is defined by:

$$
\varepsilon_{x}=\frac{A^{\prime} B^{\prime}-A B}{A B},
$$

and knowing that $A B=\mathrm{d} x^{\alpha}$, we have

$$
\varepsilon_{x}=\left(l^{\alpha-1}\right) \frac{1}{\alpha} \frac{\partial^{\alpha} u}{\partial x^{\alpha}} .
$$

Similarly, the normal strain in the $y$-direction and $z$ direction becomes

$$
\varepsilon_{y}=\left(l^{\alpha-1}\right) \frac{1}{\alpha} \frac{\partial^{\alpha} v}{\partial y^{\alpha}}, \quad \varepsilon_{z}=\frac{1}{\alpha} l^{\alpha-1} \frac{\partial^{\alpha} w}{\partial z^{\alpha}},
$$

where $l^{\alpha-1}$ is the length scale parameter similarly like in the classical non-local gradient methods and fractional 
nonlocal Kirchhoff theory [8]. The introduction of the length scale, allows finally to obtain dimensionless quantity and lets one to consider the size-dependence effects for micron and sub-micron scales.

The engineering shear strain, or the change in the angle between two originally orthogonal material lines (in this case line $A B$ and $A C$ ) is defined as:

$$
\gamma_{x y}=\frac{\pi}{2}-\angle C^{\prime} A^{\prime} B^{\prime}
$$

where $C^{\prime} A^{\prime} B^{\prime}$ is line's angle in the deformed element. For small deformations, $\alpha=\tan \alpha$ and $\beta=\tan \beta$, shear strain by neglecting higher-order terms in the displacement gradients (for small displacement gradient we have $\frac{1}{\alpha} \frac{\partial^{\alpha} u}{\partial x^{\alpha}} \ll 1$ and $\left.\frac{1}{\alpha} \frac{\partial^{\alpha} u}{\partial y^{\alpha}} \ll 1\right)$ can be then expressed as

$$
\begin{gathered}
\gamma_{x y}=\frac{\frac{1}{\alpha} \frac{\partial^{\alpha} v}{\partial x^{\alpha}} \mathrm{d} x^{\alpha}}{\mathrm{d} x^{\alpha}+\frac{1}{\alpha} \frac{\partial^{\alpha} u}{\partial x^{\alpha}} \mathrm{d} x^{\alpha}}+\frac{\frac{1}{\alpha} \frac{\partial^{\alpha} u}{\partial y^{\alpha}} \mathrm{d} y^{\alpha}}{\mathrm{d} y^{\alpha}+\frac{1}{\alpha} \frac{\partial^{\alpha} u}{\partial y^{\alpha}} \mathrm{d} y^{\alpha}}= \\
\frac{1}{\alpha} l^{\alpha-1}\left(\frac{\partial^{\alpha} v}{\partial x^{\alpha}}+\frac{\partial^{\alpha} u}{\partial y^{\alpha}}\right) .
\end{gathered}
$$

By considering similar behaviors in the $y-z$ and $x-z$ planes, these results can be easily extended to the general three-dimensional case.

Now the fractional strain-displacement relations are

$$
\begin{aligned}
& \varepsilon_{x}=\frac{1}{\alpha} l^{\alpha-1} \frac{\partial^{\alpha} u}{\partial x^{\alpha}}, \quad \varepsilon_{y}=\frac{1}{\alpha} l^{\alpha-1} \frac{\partial^{\alpha} v}{\partial y^{\alpha}}, \quad \varepsilon_{z}=\frac{1}{\alpha} l^{\alpha-1} \frac{\partial^{\alpha} w}{\partial z^{\alpha}} \\
& \gamma_{x y}=\frac{1}{\alpha} l^{\alpha-1}\left(\frac{\partial^{\alpha} v}{\partial x^{\alpha}}+\frac{\partial^{\alpha} u}{\partial y^{\alpha}}\right), \\
& \gamma_{z x}=\frac{1}{\alpha} l^{\alpha-1}\left(\frac{\partial^{\alpha} w}{\partial x^{\alpha}}+\frac{\partial^{\alpha} u}{\partial z^{\alpha}}\right), \\
& \gamma_{y z}=\frac{1}{\alpha} l^{\alpha-1}\left(\frac{\partial^{\alpha} v}{\partial z^{\alpha}}+\frac{\partial^{\alpha} w}{\partial y^{\alpha}}\right) .
\end{aligned}
$$

For completeness the two-dimensional geometric fractional strain deformation has been shown in Fig. 1.

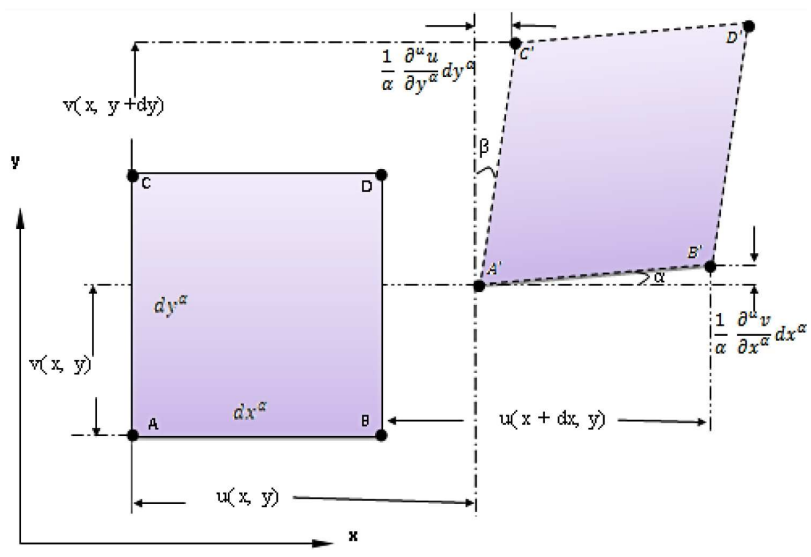

Fig. 1. Two-dimensional geometric fractional strain deformation

\subsection{Continuum view}

One considers a continuum body $B$, as the set of elements $X$ whom the reference and the current configura- tions are $R$ and $C$, respectively. The regular motion of the material body $B$ can be written as

$$
x=\aleph(X, t) \quad \text { or } \quad x_{i}=\aleph_{i}(X, t),
$$

where the mapping function $\aleph$ gives the position $x$ for each particles $X$ for all times $t$. Its inverse due to continuity assumption exists as

$$
X=N(x, t) \quad \text { or } \quad X_{A}=N_{A}(x, t),
$$

and states that the particle $X$ is located at position $x$ at time $t$. Forasmuch, as in the deformation just two stationary configurations have been considered and disregard any consideration by which the final deformed configuration is reached from the initial undeformed configuration, so the time variable is eliminated and $x=\aleph(X)$ and $X=\mathrm{N}(x)$.

The mapping function is continuous, so

$$
\mathrm{d} x_{i}=\frac{\partial \aleph_{i}(X)}{\partial X_{A}} \mathrm{~d} X_{A}=x_{i, A} \mathrm{~d} X_{A},
$$

where the deformation gradient tensor is [19]:

$$
x_{i, A} \equiv F_{i A}=\frac{\partial \aleph_{i}(X)}{\partial X_{A}}
$$

Rewriting Eq. (14) leads to the following form:

$$
\mathrm{d} x=F \cdot \mathrm{d} X .
$$

$F$ is invertible, so that the inverse $F^{-1}$ exists, therefore

$$
\mathrm{d} X=F^{-1} \cdot \mathrm{d} x, \quad F^{-1}=X_{A, i} .
$$

We can generalize the deformation gradient and its inverse as follows:

$$
\begin{aligned}
& { }^{G} F_{i A}=\frac{\partial^{\alpha} \aleph_{i}(X)}{\partial X_{A}^{\alpha}}=\frac{l^{\alpha-1}}{\alpha}|X|^{1-\alpha} \frac{\partial \aleph_{i}(X)}{\partial X_{A}}= \\
& \frac{l^{\alpha-1}}{\alpha}|X|_{A}^{1-\alpha} F_{i A}, \\
& { }^{G} F_{A i}^{-1}=\frac{\partial^{\alpha} N_{A}(X)}{\partial x_{i}^{\alpha}}=\frac{l^{\alpha-1}}{\alpha}|x|^{1-\alpha} \frac{\partial N_{A}(X)}{\partial x_{i}}= \\
& \frac{l^{\alpha-1}}{\alpha}\left|x_{i}\right|^{1-\alpha} F_{i A},
\end{aligned}
$$

where $l$ is the length scale of the isotropic material and leads to the dimensional less quantity similarly like in the non-local gradient methods.

\subsubsection{Rigid body motion}

It should be emphasized that it is crucial to observe how the fractional deformation gradients transforms under isomorphism (superimposed rigid-body motions). There is a condition for the rigid body motion, which is satisfied by classical strain.

The Lagrangian finite strain tensor is

$$
2 E=C-I \text {, }
$$

where $C=F^{T} \cdot F$ and $I$ is an identity matrix. For the classical rigid body motion

$$
C=I \rightarrow F^{T} \cdot F=I .
$$

Now, for the general form of the strains the following condition must be satisfied:

$$
{ }^{G} F^{T} \cdot{ }^{G} F=I .
$$

Two categories have been considered for value of $\alpha$ : (1) $\alpha=1$ (classical strain form), (2) $\alpha \neq 1$ (the fractional 
strain form). In the case of $\alpha=1 \mathrm{Eq}$. (20) is valid, but for $\alpha \neq 1$ :

$$
\begin{aligned}
& { }^{G} F^{T} \cdot{ }^{G} F=I \rightarrow \\
& \quad\left(\frac{l^{\alpha-1}}{\alpha}\left|X_{A}\right|^{\alpha-1}\right) F^{\mathrm{T}} \cdot\left(\frac{l^{\alpha-1}}{\alpha}\left|X_{B}\right|^{\alpha-1}\right) F=I .
\end{aligned}
$$

With respect to Eq. (20), the following condition must satisfy

$$
\begin{aligned}
& \left(\frac{l^{\alpha-1}}{\alpha}\left|X_{A}\right|^{1-\alpha}\right)=\left(\frac{l^{\alpha-1}}{\alpha}\left|X_{B}\right|^{1-\alpha}\right)=1 \rightarrow \\
& X=\alpha^{1-\alpha} l .
\end{aligned}
$$

\subsection{Fractional Euler-Bernoulli beam theory}

In the previous sections, the general form of straindisplacement relations has been obtained based on the modified CFDD. Now, these relations will be used in the one of the structural mechanics problem - beam's equations of the motion - and the FEBBT will be presented.

\subsubsection{Equation of motion}

A schematic view of a beam under distributed and axial forces has been shown in Fig. 2. Assume that its displacement field is

$$
u_{0}=-z w_{x}, \quad v_{0}=0, \quad w_{0}=w(x, t),
$$

where $u_{0}, v_{0}$ and $w_{0}$ denote the components of displacement parallel to $x-, y$ - and $z$-direction. The classical equation of motion of the Euler-Bernoulli beam is [20]:

$$
M_{x x}+f(x, t)+p w_{x x}=\rho A w_{t t}, \quad 0<x<L,
$$

where $M(x, t)=\int_{-h / 2}^{h / 2} \sigma z \mathrm{~d} z, A$ is cross-section of the beam, $\rho$ is density, $p$ and $f$ are axial and transverse forces. According to the fractional strain-displacement

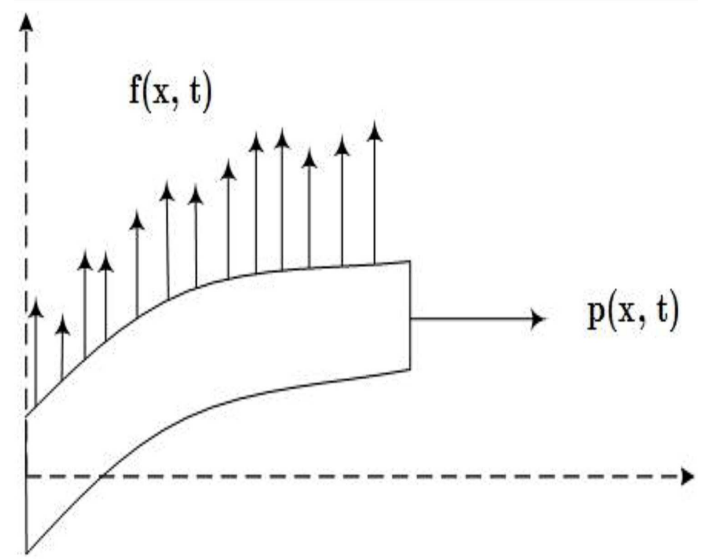

Fig. 2. A schematic view of a beam under distributed and axial forces.

relations and Eq. $(24)$ the $M(x, t)$ obtains the form as below

$$
M(x, t)=-E I\left(\frac{1}{\alpha} l^{\alpha-1} x^{1-\alpha} w_{x x}\right),
$$

where $E$ is Young's modulus, $h$ is the beam thickness and $I$ is the effective moment of inertia. Now, by substi- tuting the second derivative of Eq. (26) into Eq. (25) the fractional dynamics equation is obtained

$$
\begin{aligned}
& E I\left(\frac{1}{\alpha} l^{\alpha-1} x^{1-\alpha} w_{x x x x}\right)+E I\left(\frac{2}{\alpha} l^{\alpha-1}(1-\alpha) x^{-\alpha} w_{x x x}\right) \\
& \quad+E I\left(\frac{1}{\alpha} l^{\alpha-1}(1-\alpha)(-\alpha) x^{-1-\alpha} w_{x x}\right)+m w_{t t} \\
& \quad=p w_{x x}+f(x, t), \quad 0<x<L .
\end{aligned}
$$

For convenience, the following non-dimensional parameters are utilized:

$$
\begin{aligned}
& \hat{w}=\frac{w_{0}}{L}, \quad \hat{x}=\frac{x}{L}, \quad \hat{t}=\frac{t}{T}, \\
& T=\sqrt{\frac{\rho A L^{4}}{E I}}, \quad S_{1}=\left(\frac{1}{\alpha} l^{\alpha-1} L^{-\alpha+1}\right), \\
& S_{2}=\left(\frac{L^{2}}{E I}\right), \quad S_{3}=\frac{L^{3}}{E I} .
\end{aligned}
$$

Then the non-dimensional form of Eq. (27) is

$$
\begin{aligned}
& S_{1}\left[\hat{x}^{1-\alpha} \hat{w}_{x x x x}+2(1-\alpha) \hat{x}^{-\alpha} \hat{w}_{x x x}\right. \\
& \left.\quad+(1-\alpha)(-\alpha) \hat{x}^{-1-\alpha} \hat{w}_{x x}\right]+\hat{w}_{t t}=S_{2} p \hat{w}_{x x}+S_{3} f(\hat{x}, \hat{t}),
\end{aligned}
$$

$0<\hat{x}<1$

It should be noted that when $\alpha=1$ the classical equation of motion of the Euler-Bernoulli beam is obtained.

\subsubsection{Vibration, bending and buckling of nanobeams} based on the FEBBT

In this section, we show the effects of the fractional parameter and the length scale parameter on the free vibration, bending and buckling of nanobeams.

\subsubsection{Vibration}

In the vibration analyses, we neglect the effect of transverse loading on the beam, in the other words for analysis of the frequency of the beam under axial load, Eq. (28) reduces to the following form:

$$
\begin{aligned}
& S_{1}\left[\hat{x}^{1-\alpha} \hat{w}_{x x x x}+2(1-\alpha) \hat{x}^{-\alpha} \hat{w}_{x x x}\right. \\
& \left.\quad+(1-\alpha)(-\alpha) \hat{x}^{-1-\alpha} \hat{w}_{x x}\right]+w_{t t}-S_{2} p \hat{w}_{x x}=0 .
\end{aligned}
$$

Using modified CFDD the governing equations are easily solved by the classical numerical schemes, therefore here to calculate the non-dimensional frequency of the nanobeam we use the Galerkin weighted residual method. Based on this method we assume

$$
w(x, t) \approx \sum_{i=1}^{n} \varphi_{i}(x) q_{i}(t),
$$

where $\varphi_{i}(x)$ and $q_{i}(t)$ are the mode shape and a time dependent function to be determined, respectively. Substituting Eq. (30) into Eq. (29) yields

$$
\begin{aligned}
& S_{1}\left[\hat{x}^{1-\alpha} \sum_{i=1}^{n} \varphi_{i}^{(4)}(\hat{x}) q_{i}(\hat{t})+2(1-\alpha) \hat{x}^{-\alpha}\right. \\
& \left.\quad \times \sum_{i=1}^{n} \varphi_{i}^{\prime \prime \prime}(\hat{x}) q_{i}(\hat{t})+(1-\alpha)(-\alpha) \hat{x}^{-1-\alpha} \sum_{i=1}^{n} \varphi_{i}^{\prime \prime}(\hat{x}) q_{i}(\hat{t})\right] \\
& \quad+\sum_{i=1}^{n} \varphi_{i}(\hat{x}) \ddot{q}_{i}(\hat{t})-S_{2} p \sum_{i=1}^{n} \varphi_{i}^{\prime \prime}(\hat{x}) q_{i}(\hat{t})=\varepsilon .
\end{aligned}
$$


Next, by multiplying the result by the mode shape and integrating outcome from 0 to 1 we have

$$
M \ddot{q}(\hat{t})+K q(\hat{t})=0 .
$$

where

$$
\begin{aligned}
M & =\int_{0}^{1} \varphi_{i} \varphi_{j} \mathrm{~d} \hat{x}, \\
K & =\int_{0}^{1}\left[S _ { 1 } \left(\hat{x}^{1-\alpha} \varphi_{j}^{\prime \prime \prime \prime}+2(1-\alpha) \hat{x}^{-\alpha} \varphi_{j}^{\prime \prime \prime}\right.\right. \\
& \left.\left.+(1-\alpha)(-\alpha) \hat{x}^{-1-\alpha} \varphi_{j}^{\prime \prime}\right) \varphi_{i}+S_{2} \varphi_{j}^{\prime \prime} \varphi_{i}\right] \mathrm{d} \hat{x} .
\end{aligned}
$$

The non-dimensional eigenproblem of the beam is then

$$
\left[M \omega^{2}+S_{1} K\right] \bar{q}=0 .
$$

\subsubsection{Bending}

The static deflection is obtained from Eq. (28) by setting axial force and the inertial term to zero as below

$$
\begin{aligned}
& S_{1}\left[\hat{x}^{1-\alpha} \hat{w}_{x x x x}+2(1-\alpha) \hat{x}^{-\alpha} \hat{w}_{x x x}\right. \\
& \left.\quad+(1-\alpha)(-\alpha) \hat{x}^{-1-\alpha} \hat{w}_{x x}\right]=S_{3} f(\hat{x}, \hat{t}) .
\end{aligned}
$$

To solve Eq. (35) the Galerkin method is used again. The transverse displacement of the beam is as below

$$
w(x) \approx \sum_{i=1}^{n} a_{i} \varphi_{i}(x) .
$$

Substituting Eq. (36) into Eq. (35) one has

$$
\begin{aligned}
& S_{1}\left[\hat{x}^{1-\alpha} \sum_{i=1}^{n} \varphi_{i}^{(4)}(\hat{x}) q_{i}(t)+2(1-\alpha) \hat{x}^{-\alpha}\right. \\
& \times \sum_{i=1}^{n} \varphi_{i}^{\prime \prime \prime}(\hat{x}) q_{i}(t)+(1-\alpha)(-\alpha) \hat{x}^{-1-\alpha} \\
& \left.\quad \times \sum_{i=1}^{n} \varphi_{i}^{\prime \prime}(\hat{x}) q_{i}(t)\right]=S_{3} f(\hat{x}, \hat{t}) .
\end{aligned}
$$

Multiplying Eq. (37) by $\varphi_{i}$, as a weight function in the Galerkin method, and integrating the outcomes from $x=0$ to 1 , the set of algebraic equations is obtained (Eq. (38)). By solution of these algebraic equations, the deflection of nanobeam can be determined in a form

$$
F_{i}=\sum_{j=1}^{n} K_{i j} a_{j}, i=1,2, \ldots, n,
$$

where

$$
\begin{aligned}
K_{i j} & =S_{1} \int_{0}^{1}\left(\hat{x}^{1-\alpha} \varphi_{j}^{\prime \prime \prime \prime}+2(1-\alpha) \hat{x}^{-\alpha} \varphi_{j}^{\prime \prime \prime}\right. \\
& \left.+(1-\alpha)(-\alpha) \hat{x}^{-1-\alpha} \varphi_{j}^{\prime \prime}\right) \varphi_{i} \mathrm{~d} \hat{x}, \\
F_{i} & =\int_{0}^{1} S_{3} f(x, t) \varphi_{i} \mathrm{~d} \hat{x} .
\end{aligned}
$$

\subsubsection{Buckling}

The buckling load is obtained from Eq. (28) by setting the transverse force and the inertia term to zero, so the governing equation is

$$
\begin{aligned}
& S_{1}\left[\hat{x}^{1-\alpha} \hat{w}_{x x x x}+2(1-\alpha) \hat{x}^{-\alpha} \hat{w}_{x x x}\right. \\
& \left.\quad+(1-\alpha)(-\alpha) \hat{x}^{-1-\alpha} \hat{w}_{x x}\right]=S_{2} p \hat{w}_{x x} .
\end{aligned}
$$

Next, by assuming the axial force $p=-p_{c r}$ and applying the Galerkin method, the buckling load reads

$$
p_{c r}=\frac{K^{f}}{K},
$$

where

$$
\begin{aligned}
K^{f} & =S_{1} \int_{0}^{1}\left(\hat{x}^{1-\alpha} \varphi_{j}^{\prime \prime \prime \prime}+2(1-\alpha) \hat{x}^{-\alpha} \varphi_{j}^{\prime \prime \prime}\right. \\
& \left.+(1-\alpha)(-\alpha) \hat{x}^{-1-\alpha} \varphi_{j}^{\prime \prime}\right) \varphi_{i} \mathrm{~d} \hat{x}, \\
K & =S_{2} \int_{0}^{1} \varphi_{j}^{\prime \prime} \varphi_{i} \mathrm{~d} \hat{x} .
\end{aligned}
$$

\subsubsection{Results}

This part contains two sections. In the first section, to demonstrate the functionality of FEBBT, the nondimensional frequency of micro $\mathrm{C}-\mathrm{C}$ beams under axial force has been studied and in the second section, the effects of the fractional and the non-dimensional length scale parameters have been shown on the free vibration, bending and buckling of nanobeams. It is important that the results of Sect. 3.3.3.1 are contrasted with the experimental data [20].

3.3.3.1. Is the FEBBT is more efficient than the EulerBernoulli theory (EBT)?

In this section, to validate our fractional theory and to show that FEBBT is more appropriate than EBT, the vibration of $\mathrm{C}-\mathrm{C}$ micro beams under axial force has been studied. A comparison has been done between experimental results [21] and the FEBBT and EBT. In Table I the physical properties of the microbeam have been shown and the value of axial force is $0.0009 \mathrm{~N}$.

Geometrical and physical proper-

TABLE I ties of the micro $\mathrm{C}-\mathrm{C}$ beams

\begin{tabular}{l|c}
\hline \hline Property & Value \\
\hline length $[\mu \mathrm{m}]$ & $210,310,410,510$ \\
width $[\mu \mathrm{m}]$ & 100 \\
height $[\mu \mathrm{m}]$ & 1.5 \\
Young modulus $[\mathrm{GPa}]$ & $E /(1-v)=166$ \\
density $\left[\mathrm{kg} / \mathrm{m}^{3}\right]$ & $\rho=2329$
\end{tabular}

The fractional parameter and the length scale parameter give us the ability to describe the behavior of materials better than the classical EBT. Figure 3 shows the effects of different length scale parameter $(\lambda=l / L)$ on the nondimensional frequency for $L=210 \mu \mathrm{m}$ when $0<\alpha \leq 1$. For $\lambda=0.2, \lambda=0.4$ and $\lambda=0.6$ decreasing $\alpha$ from 1 to 0.1 causes the increase in the non-dimensional frequency. Table II shows the calculated non-dimensional frequency of different microbeams based on FEBBTs and EBT. 
TABLE II

The compression of the non-dimensional frequency of C-C micro beams based on the FEBBT and EBT with experimental data [20] $(p=0.0009 \mathrm{~N}, \lambda=1$, error $=$ absolute number of (experimental value - calculated value $))$

\begin{tabular}{l|c|c|c|c|c|c|c|c|c|c|c|c|c}
\hline \hline & \multicolumn{10}{|c|}{$\alpha$ (fractional parameter) } & Experimental \\
\cline { 2 - 13 } & 0.89 & 0.90 & 0.91 & 0.92 & 0.93 & 0.94 & 0.95 & 0.96 & 0.97 & 0.98 & 0.99 & $1($ EBT) & {$[20]$} \\
\hline$L=210$ & 322.74 & 322.73 & 322.73 & 322.76 & 322.83 & 322.95 & 323.11 & 323.31 & 323.55 & 323.84 & 324.17 & 324.55 & 322.05 \\
error1 & 0.69 & 0.68 & 0.68 & 0.71 & 0.78 & 0.90 & 1.06 & 1.26 & 1.50 & 1.79 & 2.12 & 2.50 & - \\
$L=310$ & 162.65 & 162.65 & 162.64 & 162.66 & 162.69 & 162.74 & 162.80 & 162.89 & 162.99 & 163.11 & 163.25 & 163.41 & 163.22 \\
error2 & 0.57 & 0.57 & 0.58 & 0.56 & 0.53 & 0.48 & 0.42 & 0.33 & 0.23 & 0.11 & 0.03 & 0.19 & - \\
$L=410$ & 103.41 & 103.40 & 103.40 & 103.40 & 103.42 & 103.45 & 103.48 & 103.52 & 103.58 & 103.64 & 103.71 & 103.79 & 102.17 \\
error3 & 1.24 & 1.23 & 1.23 & 1.23 & 1.25 & 1.28 & 1.31 & 1.35 & 1.41 & 1.47 & 1.54 & 1.62 & - \\
$L=510$ & 74.55 & 74.55 & 74.55 & 74.55 & 74.56 & 74.58 & 74.59 & 74.62 & 74.65 & 74.69 & 74.73 & 74.77 & 73.79 \\
error4 & 0.76 & 0.76 & 0.76 & 0.76 & 0.77 & 0.79 & 0.80 & 0.83 & 0.86 & 0.90 & 0.94 & 0.98 & - \\
\hline$\sum_{1}^{4}$ error & 3.26 & 3.24 & 3.24 & 3.27 & 3.34 & 3.44 & 3.59 & 3.77 & 4.00 & 4.26 & 4.62 & 5.29 & -
\end{tabular}

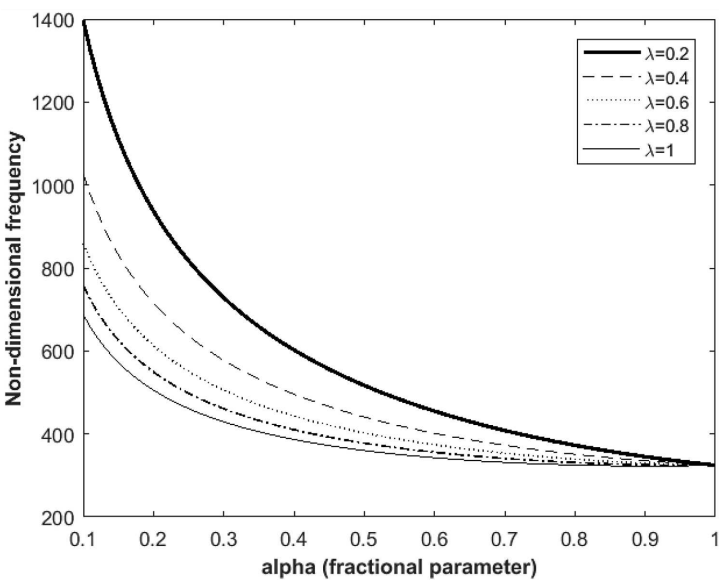

Fig. 3. Effects of fractional parameter $\alpha$ and length scale parameter $\lambda$ on the non-dimensional frequency $\omega_{1}$ of fractional Euler-Bernoulli micro C-C nanobeam $(p=$ $0.0009 \mathrm{~N}, L=210 \mu \mathrm{m})$

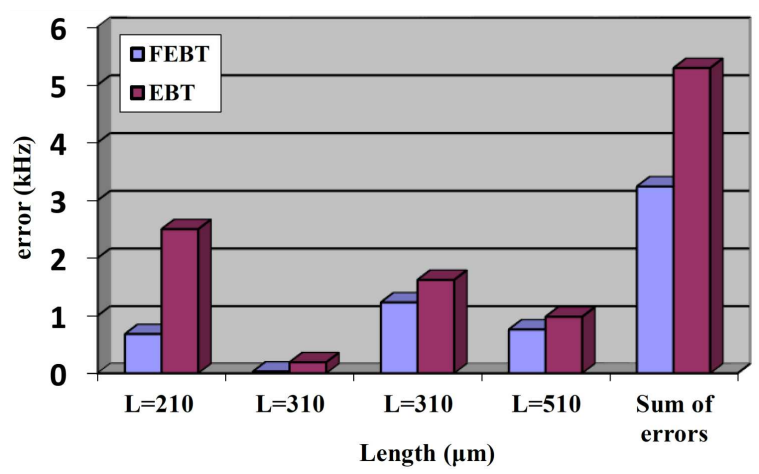

Fig. 4. The compression of calculated the errors of the FEBBT and EBT. Blue columns show error of FEBBT and red columns show the error of EBT.

The errors are the difference between experimentally measured frequencies and the calculated ones. It is visible from Table II that the errors of FEBBT are lower than the errors of EBT. As it can be seen, FEBBT reduces the sum of the error from $5.29 \mathrm{kHz}$ to $3.24 \mathrm{kHz}$. In addition, FEBBT reduces the error for each lengths, for instance in $L=210 \mu \mathrm{m}$ FEBBT reduces the error from $2.5 \mathrm{kHz}$ to $0.68 \mathrm{kHz}$, for $L=310 \mu \mathrm{m}$ from $0.19 \mathrm{kHz}$ to $0.03 \mathrm{kHz}$, for $L=410 \mu \mathrm{m}$ from $1.62 \mathrm{kHz}$ to $1.23 \mathrm{kHz}$ and for $L=510 \mu \mathrm{m}$ from $0.98 \mathrm{kHz}$ to $0.76 \mathrm{kHz}$. In Fig. 4, the error of the calculated non-dimensional frequencies based on FEBBT and EBT has been compared. In Fig. 3 and Table II, the values of errors show that FEBBT could be more efficient than EBT.

3.3.3.2. Numerical results for free vibration, bending, and buckling of nanobeams

In this section, the effects of the fractional parameter $\alpha$ and the length scale parameter $\lambda$ on the non-dimensional frequency, non-dimensional center deflection and nondimensional buckling load of a simply supported nano beam have been shown.

Free vibration of nano beam means the axial force is zero $(p=0)$. In Table 3 and in Fig. 5 , the effects of the fractional parameter $\alpha$ and length scale parameter $\lambda$ have been shown on the first, second and third nondimensional frequency of simply supported beam. As it can be seen from Table III and Fig. 4, decreasing of the fractional parameter from 1 to 0.1 leads to the increase of the first, the second and the third non-dimensional natural frequencies. This increase for $\omega_{3}$ is larger than for $\omega_{2}$ and $\omega_{1}$ and for $\omega_{2}$ is more than the $\omega_{1}$. In addition, it can be seen that at the constant $\alpha$ the increase of $\lambda$ causes the decrease in the natural frequencies.

The non-dimensional center deflection of the simply supported nanobeam has been shown in Table IV for different values of $\lambda$ and $\alpha$, when $L / h$ has different values. It is visible that for different $L / h$ ratios when $\alpha$ decreases from 1 to 0.1 the maximum non-dimensional deflection decreases and this means that decrease of $\alpha$ increases the stiffness of simply supported beams. In Fig. 6 , the variation of the center deflection versus $\alpha$ for 5 values of $\lambda$ has been shown when $L / h=50$. At the constant value of $\alpha$, increase of $\lambda$ causes the increase in the center deflection, so one can be said that it causes the decrease in the beam stiffness, and it can be seen also that the variation of the non-dimensional center deflection increases as the value of $\lambda$ decreases. 
Non-dimensional natural frequency of simply supported nanobeam $(L=10 \mathrm{~nm}, b=h=0.1 L, \rho=1, \lambda=l / L)$

TABLE III

\begin{tabular}{c|c|c|c|c|c|c|c|c|c|c|c}
\hline \hline $\begin{array}{c}\text { Length } \\
\text { scale }\end{array}$ & Frequency & $\alpha=0.1$ & $\alpha=0.2$ & $\alpha=0.3$ & $\alpha=0.4$ & $\alpha=0.5$ & $\alpha=0.6$ & $\alpha=0.7$ & $\alpha=0.8$ & $\alpha=0.9$ & $\begin{array}{c}\alpha=1 \\
\text { (classical } \\
\text { theory) }\end{array}$ \\
\hline \multirow{3}{*}{$\lambda=0.2$} & $\omega_{1}$ & 46.99 & 31.66 & 24.64 & 20.36 & 17.39 & 15.16 & 13.42 & 12.02 & 10.85 & 9.87 \\
& $\omega_{2}$ & 187.23 & 125.73 & 97.64 & 80.54 & 68.72 & 59.95 & 53.13 & 47.67 & 43.20 & 39.48 \\
& $\omega_{3}$ & 420.86 & 282.39 & 219.13 & 180.65 & 154.09 & 134.40 & 119.15 & 106.96 & 97.03 & 88.83 \\
\hline \multirow{3}{*}{$\lambda=0.4$} & $\omega_{1}$ & 34.40 & 23.99 & 19.34 & 16.54 & 14.62 & 13.20 & 12.10 & 11.21 & 10.48 & 9.87 \\
& $\omega_{2}$ & 137.06 & 95.29 & 76.60 & 65.42 & 57.79 & 52.19 & 47.88 & 44.48 & 41.72 & 39.48 \\
& $\omega_{3}$ & 308.09 & 214.01 & 171.92 & 146.73 & 129.57 & 117.00 & 107.38 & 99.80 & 93.73 & 88.83 \\
\hline \multirow{3}{*}{$\lambda=0.6$} & $\omega_{1}$ & 28.66 & 20.40 & 16.78 & 14.64 & 13.21 & 12.17 & 11.38 & 10.77 & 10.27 & 9.87 \\
& $\omega_{2}$ & 114.20 & 81.02 & 66.47 & 57.92 & 52.22 & 48.12 & 45.06 & 42.71 & 40.89 & 39.48 \\
& $\omega_{3}$ & 256.71 & 181.97 & 149.18 & 129.93 & 117.08 & 107.89 & 101.04 & 95.83 & 91.85 & 88.83 \\
\hline \multirow{3}{*}{$\lambda=0.8$} & $\omega_{1}$ & 25.18 & 18.18 & 15.17 & 13.43 & 12.29 & 11.49 & 10.90 & 10.46 & 10.12 & 9.87 \\
& $\omega_{2}$ & 100.33 & 72.22 & 60.10 & 53.13 & 48.59 & 45.43 & 43.15 & 41.50 & 40.30 & 39.48 \\
& $\omega_{3}$ & 225.53 & 162.19 & 134.89 & 119.18 & 108.96 & 101.86 & 96.78 & 93.12 & 90.53 & 88.83 \\
\hline \multirow{3}{*}{$\lambda=1$} & $\omega_{1}$ & 22.77 & 16.63 & 14.03 & 12.56 & 11.63 & 10.99 & 10.54 & 10.23 & 10.01 & 9.87 \\
& $\omega_{2}$ & 90.75 & 66.05 & 55.59 & 49.69 & 45.96 & 43.45 & 41.73 & 40.58 & 39.86 & 39.48 \\
& $\omega_{3}$ & 203.99 & 148.34 & 124.76 & 111.47 & 103.04 & 97.41 & 93.59 & 91.06 & 89.53 & 88.83
\end{tabular}

Center deflection of simply supported nanobeam subjected to uniform load $\bar{f}(L=10 \mathrm{~nm}, E=169 \mathrm{GPa}$,

TABLE IV $\bar{f}=10, \lambda=l / L)$

\begin{tabular}{c|c|c|c|c|c|c|c|c|c|c|c}
\hline \hline \multirow{2}{*}{$L / h$} & $\begin{array}{c}\text { Length } \\
\text { scale }\end{array}$ & $\alpha=0.1$ & $\alpha=0.2$ & $\alpha=0.3$ & $\alpha=0.4$ & $\alpha=0.5$ & $\alpha=0.6$ & $\alpha=0.7$ & $\alpha=0.8$ & $\alpha=0.9 \begin{array}{c}\alpha=1 \\
\text { (classical } \\
\text { theory) }\end{array}$ \\
\hline \multirow{5}{*}{100} & $\lambda=0.2$ & 0.474 & 1.011 & 1.622 & 2.318 & 3.114 & 4.027 & 5.074 & 6.276 & 7.657 & 9.243 \\
& $\lambda=0.4$ & 0.885 & 1.761 & 2.635 & 3.513 & 4.404 & 5.313 & 6.246 & 7.209 & 8.207 & 9.243 \\
& $\lambda=0.6$ & 1.274 & 2.435 & 3.499 & 4.481 & 5.394 & 6.249 & 7.054 & 7.818 & 8.546 & 9.243 \\
& $\lambda=0.8$ & 1.651 & 3.065 & 4.280 & 5.325 & 6.229 & 7.011 & 7.690 & 8.281 & 8.796 & 9.243 \\
& $\lambda=1$ & 2.018 & 3.664 & 5.003 & 6.088 & 6.964 & 7.665 & 8.223 & 8.659 & 8.994 & 9.243 \\
\hline \multirow{5}{*}{75} & $\lambda=0.2$ & 0.150 & 0.320 & 0.513 & 0.733 & 0.985 & 1.274 & 1.605 & 1.986 & 2.423 & 2.924 \\
& $\lambda=0.4$ & 0.280 & 0.557 & 0.834 & 1.112 & 1.394 & 1.681 & 1.976 & 2.281 & 2.597 & 2.924 \\
& $\lambda=0.6$ & 0.403 & 0.770 & 1.107 & 1.418 & 1.707 & 1.977 & 2.232 & 2.474 & 2.704 & 2.924 \\
& $\lambda=0.8$ & 0.522 & 0.970 & 1.354 & 1.685 & 1.971 & 2.218 & 2.433 & 2.620 & 2.783 & 2.924 \\
& $\lambda=1$ & 0.639 & 1.159 & 1.583 & 1.926 & 2.203 & 2.425 & 2.602 & 2.740 & 2.846 & 2.924 \\
\hline \multirow{5}{*}{50} & $\lambda=0.2$ & 0.030 & 0.063 & 0.101 & 0.145 & 0.195 & 0.252 & 0.317 & 0.392 & 0.479 & 0.578 \\
& $\lambda=0.4$ & 0.055 & 0.110 & 0.165 & 0.220 & 0.275 & 0.332 & 0.390 & 0.451 & 0.513 & 0.578 \\
& $\lambda=0.6$ & 0.080 & 0.152 & 0.219 & 0.280 & 0.337 & 0.391 & 0.441 & 0.489 & 0.534 & 0.578 \\
& $\lambda=0.8$ & 0.103 & 0.192 & 0.267 & 0.333 & 0.389 & 0.438 & 0.481 & 0.518 & 0.550 & 0.578 \\
& $\lambda=1$ & 0.126 & 0.229 & 0.313 & 0.381 & 0.435 & 0.479 & 0.514 & 0.541 & 0.562 & 0.578 \\
\hline \multirow{3}{*}{30} & $\lambda=0.2$ & 0.004 & 0.008 & 0.013 & 0.019 & 0.025 & 0.033 & 0.041 & 0.051 & 0.062 & 0.075 \\
& $\lambda=0.4$ & 0.007 & 0.014 & 0.021 & 0.028 & 0.036 & 0.043 & 0.051 & 0.058 & 0.066 & 0.075 \\
& $\lambda=0.6$ & 0.010 & 0.020 & 0.028 & 0.036 & 0.044 & 0.051 & 0.057 & 0.063 & 0.069 & 0.075 \\
& $\lambda=0.8$ & 0.013 & 0.025 & 0.035 & 0.043 & 0.050 & 0.057 & 0.062 & 0.067 & 0.071 & 0.075 \\
& $\lambda=1$ & 0.016 & 0.030 & 0.041 & 0.049 & 0.056 & 0.062 & 0.067 & 0.070 & 0.073 & 0.075
\end{tabular}
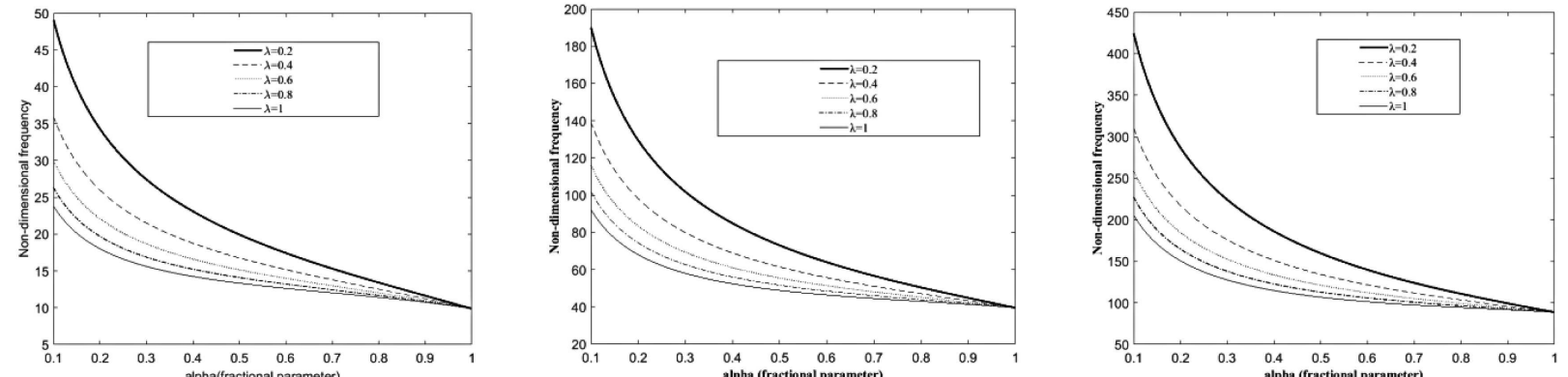

Fig. 5. The effects of the fractional parameter $(\alpha)$ and the length scale parameter $(\lambda)$ on the non-dimensional frequency of the fractional Euler-Bernoulli simply supported nanobeam. From left: (1) The first non-dimensional frequency $\left(\omega_{1}\right)$, $(2)$ the second non-dimensional frequency $\left(\omega_{2}\right),(3)$ the third non-dimensional frequency $\left(\omega_{3}\right)$. 
Non-dimensional critical buckling load $\left(\bar{p}_{c r}=p_{c r}\left(\frac{L^{2}}{E I}\right)\right)$ of simply supported nanobeam $(L=10 \mathrm{~nm}$,

$b=h=0.1 L, \lambda=l / L)$

\begin{tabular}{c|c|c|c|c|c|c|c|c|c|c}
\hline \hline $\begin{array}{c}\text { Length } \\
\text { scale }\end{array}$ & $\alpha=0.1$ & $\alpha=0.2$ & $\alpha=0.3$ & $\alpha=0.4$ & $\alpha=0.5$ & $\alpha=0.6$ & $\alpha=0.7$ & $\alpha=0.8$ & $\alpha=0.9$ & $\begin{array}{c}\alpha=1 \\
\text { (classical } \\
\text { theory) }\end{array}$ \\
\hline$\lambda=0.1$ & 417.461 & 176.797 & 99.961 & 63.669 & 43.316 & 30.742 & 22.476 & 16.802 & 12.784 & 9.870 \\
$\lambda=0.2$ & 223.712 & 101.543 & 61.533 & 42.006 & 30.629 & 23.298 & 18.256 & 14.627 & 11.927 & 9.870 \\
$\lambda=0.3$ & 155.313 & 73.414 & 46.328 & 32.935 & 25.009 & 19.810 & 16.165 & 13.488 & 11.454 & 9.870 \\
$\lambda=0.4$ & 119.884 & 58.321 & 37.878 & 27.713 & 21.658 & 17.657 & 14.829 & 12.734 & 11.129 & 9.870 \\
$\lambda=0.5$ & 98.072 & 48.786 & 32.401 & 24.241 & 19.372 & 16.149 & 13.868 & 12.178 & 10.883 & 9.870 \\
$\lambda=0.6$ & 83.230 & 42.165 & 28.518 & 21.729 & 17.684 & 15.013 & 13.130 & 11.742 & 10.687 & 9.870 \\
$\lambda=0.7$ & 72.448 & 37.273 & 25.601 & 19.809 & 16.372 & 14.116 & 12.537 & 11.385 & 10.523 & 9.870 \\
$\lambda=0.8$ & 64.244 & 33.497 & 23.317 & 18.284 & 15.315 & 13.381 & 12.045 & 11.085 & 10.383 & 9.870 \\
$\lambda=0.9$ & 57.783 & 30.485 & 21.471 & 17.036 & 14.439 & 12.766 & 11.626 & 10.827 & 10.262 & 9.870 \\
$\lambda=1$ & 52.555 & 28.020 & 19.945 & 15.993 & 13.698 & 12.239 & 11.265 & 10.601 & 10.154 & 9.870
\end{tabular}

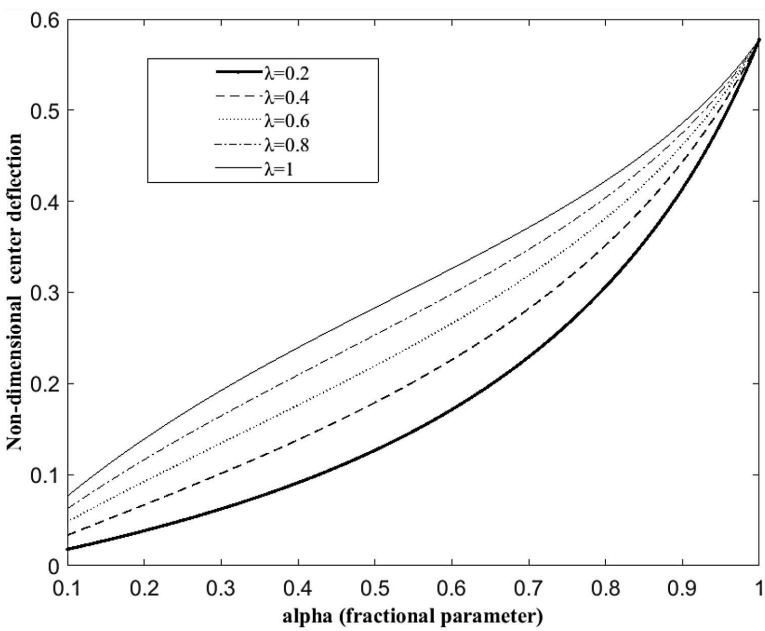

Fig. 6. The effects of the fractional parameter $\alpha$ and the length scale parameter $\lambda$ on the non-dimensional center deflection of the fractional Euler-Bernoulli simply supported nanobeam $(L / h=50)$.

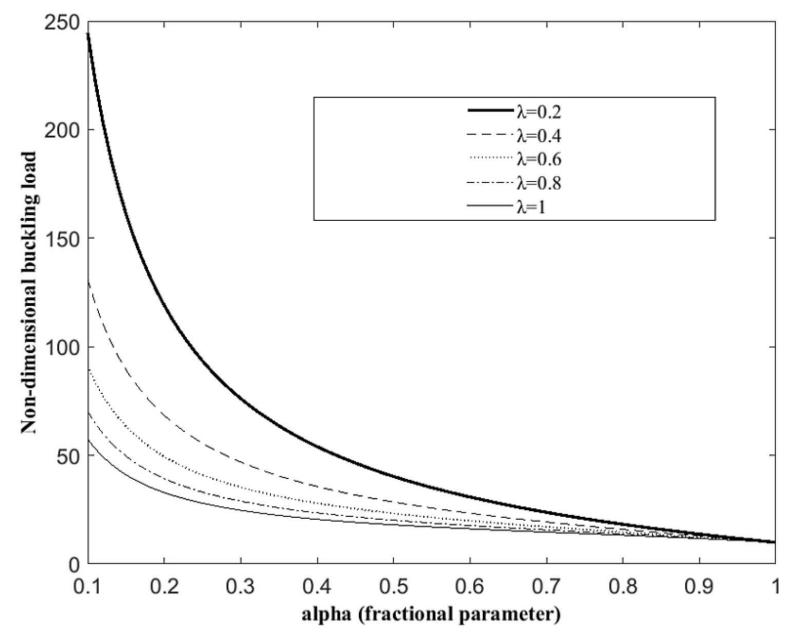

Fig. 7. The effects of the fractional parameter $\alpha$ and the length scale parameter $\lambda$ on the non-dimensional buckling load of the fractional Euler-Bernoulli simply supported nanobeam $(L / h=100)$.
Finally, in Table $\mathrm{V}$ the non-dimensional buckling load has been shown based on FEBBT and EBT when $L / h=$ 100. The non-dimensional buckling loads calculated by the EBT are smaller than those one calculated by the FEBBT. From Table V, decrease of $\alpha$ from 1 to 0.1 causes the decrease in the non-dimensional buckling load and when $\alpha$ is constant the increase of $\lambda$ causes decrease of buckling load. In Fig. 7, the non-dimensional buckling load versus $\alpha$ for different values of $\lambda$ has been shown. As it can be seen, the variation of the non-dimensional buckling load increases as the value of $\lambda$ decreases.

\section{Conclusion}

The general form of the strain and the fractional deformation gradient has been presented based on the modified CFDD. Its formulation has been studied based on two concepts: (1) the geometrical - by using fractional Taylor series expansion method and (2) the continuum mechanics' view.

The presented FEBBT is based on this general form of strain. The FEBBT two additional free parameters appear: the fractional parameter to control the order of the displacement in constitutive relation; and the length scale parameter to consider the size effects in micron and submicron scales. To show that that FEBBT can be more efficient than classical EBT the vibration of micro C-C beams under axial force was studied and it was shown that the errors of the calculated non-dimensional frequencies compared to the experimental evidence is smaller for the FEBBT compared to the results obtained by EBT.

Finally, the effects of the fractional parameter and the length scale parameter were studied on the free vibration, bending and buckling of simply supported nanobeam. It appeared that when the length scale parameter is constant, decrease of the fractional parameter from 1 to 0.1 increases both the natural frequencies, the buckling load, and decreases the maximum deflection of nanobeam. On the other hand, when the fractional parameter is constant, the increase of the non-dimensional length scale from 0 to 1 decreases both the natural frequency and the buckling load, and increases center deflection of the nanobeam. 


\section{Acknowledgments}

This work is supported by the National Science Centre, Poland under grant No. 2017/27/B/ST8/00351.

\section{Appendix A}

Conformable fractional derivatives for multi-variables function:

Assume the function $f(x, t)$, we have

$f_{x}(x, y)=\frac{\mathrm{d} f(x, y)}{\mathrm{d} x}=\lim _{h \rightarrow 0} \frac{f(x+h, y)-f(x, y)}{h}$,

$f_{y}(x, y)=\frac{\mathrm{d} f(x, y)}{\mathrm{d} y}=\lim _{h \rightarrow 0} \frac{f(x, y+h)-f(x, y)}{h}$.

Based on modified CFDD we have

$$
\begin{aligned}
& f_{x}^{\alpha}(x, y)=\frac{\mathrm{d}^{\alpha} f(x, y)}{\mathrm{d} x^{\alpha}}= \\
& \lim _{\varepsilon \rightarrow 0} \frac{f^{(\lceil\alpha\rceil-1)}\left(x+\varepsilon x^{(\lceil\alpha\rceil-\alpha)}, y\right)-f^{(\lceil\alpha\rceil-1)}(x, y)}{\varepsilon}, \\
& f_{y}^{\alpha}(x, y)=\frac{\mathrm{d}^{\alpha} f(x, y)}{\mathrm{d} y^{\alpha}}= \\
& \lim _{\varepsilon \rightarrow 0} \frac{f^{(\lceil\alpha\rceil-1)}\left(x, y+\varepsilon y^{(\lceil\alpha\rceil-1)}\right)-f^{(\lceil\alpha\rceil-1)}(x, y)}{\varepsilon} .
\end{aligned}
$$

If $0<\alpha \leq 1$, let $h=\varepsilon|x|^{\alpha-1}, h=\varepsilon|y|^{\alpha-1}$ then Eq. (A2) is

$$
\begin{gathered}
f_{x}^{\alpha}(x, y)=\frac{\mathrm{d}^{\alpha} f(x, y)}{\mathrm{d} x^{\alpha}}= \\
\lim _{\varepsilon \rightarrow 0} \frac{f^{(\lceil\alpha\rceil-1)}\left(x+\varepsilon|x|^{(\lceil\alpha\rceil-1)}, y\right)-f^{(\lceil\alpha\rceil-1)}(x, y)}{\varepsilon}= \\
|x|^{1-\alpha} \lim _{h \rightarrow 0} \frac{f(x+h, y)-f(x, y)}{h}=|x|^{1-\alpha} \frac{\mathrm{d} f(x, y)}{\mathrm{d} x}, \\
f_{y}^{\alpha}(x, y)=\frac{\mathrm{d}^{\alpha} f(x, y)}{\mathrm{d} y^{\alpha}}= \\
\lim _{\varepsilon \rightarrow 0} \frac{f^{(\lceil\alpha\rceil-1)}\left(x, y+\varepsilon|y|^{(\lceil\alpha\rceil-1)}\right)-f^{(\lceil\alpha\rceil-1)}(x, y)}{\varepsilon}= \\
|y|^{1-\alpha} \lim _{h \rightarrow 0} \frac{f(x, y)-f(x, y+h)}{h}=|y|^{1-\alpha} \frac{\mathrm{d} f(x, y)}{\mathrm{d} y} .
\end{gathered}
$$

[14] Z. Rahimi, W. Sumelka, X.J. Yang, Europ. Phys. J. Plus 132, 479 (2017).

[15] R. Khalil, M. Al Horani, A. Yousef, M. Sababheh, J. Computat. Appl. Math. 264, 65 (2014).

[16] A. Tallafha, S. Al Hihi, Int. J. Pure Appl. Math. 107, 1037 (2016).

[17] T. Abdeljawad, "On conformable fractional calculus", arXiv preprint, arXiv:1402.6892 (2014).

[18] W. Sumelka, J. Therm. Stresses 37, 678 (2014).

[19] G.T. Mase, R.E. Smelser, G.E. Mase, Continuum Mechanics for Engineers, CRC Press, 2011.

[20] L. Meirovitch, R.G. Parker, Appl. Mech. Rev. 54 B100 (2001).

[21] H.A. Tilmans, R. Legtenberg, Sensors Actuat. A Phys. 45, 67 (1994) 